\title{
X Congreso Ibérico de Agroingeniería

\section{Análisis de las diferencias en la elección de valor económico de árboles urbanos de Madrid cuando se muestran al natural y en fotografía}

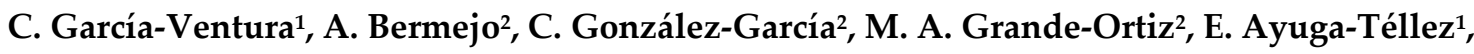
A. Sánchez de Medina-Garrido ${ }^{1}$

1 Buildings, Infrastructures and Projects for Rural and Environmental Engineering (BIPREE), Universidad Politécnica de Madrid; esperanza.ayuga@upm.es; claudia.gventura@upm.es; alvaro.sanchezdemedina@upm.es

2 Departamento de Ingeniería y Gestión Forestal y Ambiental, ETSI de Montes, Universidad Politécnica de Madrid; alfonso.bersanz@gmail.com; m.angeles.grande@upm.es; concepcion.gonzalez@upm.es

Resumen: Conocer el valor económico de un bien siempre ha sido un paso importante para poder mejorar su gestión. La Comunidad de Madrid legisló la aplicación de la primera versión de la Norma Granada como método de valoración del arbolado ornamental de su territorio. Sin embargo, los árboles de la ciudad de Madrid no cuentan con esta tasación. En los estudios de valoración de bienes socio-ambientales con participación pública, como arbolado o paisajes, se utiliza en numerosas ocasiones la fotografía del bien que se va a valorar. En el caso del arbolado, donde su valor está muy relacionado con el tamaño del árbol y con muchas características que se aprecian a la vista del ejemplar, es importante verificar la validez de las fotografías como medio para obtener las valoraciones del público. En este trabajo se va a realizar una comparación entre las tasaciones elegidas por los mismos ciudadanos al ver el árbol al natural y al verlo en fotografía. Los métodos de tasación que se han utilizado son: el método americano, Council of Tree Landscape Appraisers (CTLA), la Norma Granada y el método Burnley. Se emplearon 10 ejemplares de 10 especies diferentes y se realizaron 10 encuestas por pie. En total 100 encuestas por cada especie de árbol, que hacen un total de 1000 encuestas realizadas. Las encuestas fueron realizadas de manera aleatoria e individual. Cada persona encuestada eligió uno de los tres valores que se le mostraron, tanto al ver el ejemplar en fotografía como al verlo al natural. El 83,9\% de las 1000 encuestas dieron como resultado la misma elección de valor viendo el ejemplar "in situ" y viéndolo en fotografía.

Palabras clave: tasación, verde urbano, encuesta de opinión,

\section{Introducción}

La ciudad de Madrid ofrece un patrimonio verde de gran extensión y diversidad dotada de más de 1,5 millones de árboles [1] que constituyen un auténtico patrimonio vegetal.

Cuando no se conoce el valor monetario de los recursos naturales las actuaciones y normas que se ocupan de problemas ambientales pueden resultar insuficientes. Saber el valor de un bien siempre permite mejorar su gestión. Los métodos de valoración del arbolado urbano aportan una referencia económica o monetaria del beneficio que éste ofrece al ciudadano. También deben reflejar factores como el valor del suelo en el que se ubican, importancia histórica, calidad, beneficios sociales y medioambientales, y los costes de mantenimiento que conllevan. 


\section{CONGRESO IBÉRICO DE AGROINGENIERÍA \\ X CONGRESSO IBÉRICO DE AGROENGENHARIA \\ 3 - 6 septiembre 2019, Huesca - España}

Por tanto, la valoración tiene que ser una de las bases que se deben emplear para la toma de decisiones en su gestión. En otras palabras, hay que considerar la valoración como un instrumento para la administración y para la propia sociedad.

En 1991 la Comunidad de Madrid implantó en la normativa al respecto la aplicación de la versión de la Norma Granada, vigente en aquel momento, como método de valoración del arbolado ornamental en todo su territorio. Sin embargo, los árboles de la ciudad de Madrid son bienes que, actualmente, no cuentan con la tasación municipal que permita a los usuarios conocer el valor económico de los ejemplares. Sólo en el caso de alguna infracción que requiera la tasación del bien, o de solicitud de permisos de intervención, se tasa el ejemplar con dicha Norma. Recientemente se ha publicado una valoración mediante el modelo i-Tree que estima globalmente el valor del bosque urbano de Madrid, incluyendo el Monte del Pardo y Soto de Viñuelas [2].

La valoración del arbolado urbano no se caracteriza por ser precisa, ya que depende del objetivo de la valoración y del nivel de experiencia del evaluador [3]. En la mayoría de los "métodos comúnmente utilizados", el valor se define monetariamente con base en la percepción que el "experto" tiene del árbol, que para estos propósitos, la valoración pasa por establecer un criterio mensurable y objetivo, considerando aspectos o variables tales como si se encuentra solo o en grupo, el deterioro físico, especie y variedad, tamaño, edad, estado y ubicación, entre otras variables, que pueden llegar a intervenir [4].

En los estudios de valoración de bienes socio-ambientales con participación pública, como arbolado o paisajes, se utiliza en numerosas ocasiones la fotografía del bien que se va a valorar [5, 6, 7 y 8]. En el caso del arbolado, donde su valor está muy relacionado con el tamaño del árbol y con muchas características que se aprecian a la vista del ejemplar, el número de estudios a través de fotografías es menor [8].

Así, se han realizado estudios de paisaje [9] en Devon (UK) y Asturias (España), comparando las opiniones de visitas a los espacios "in situ" con las fotografías de los mismos sitios, encontrando un alto grado de consenso en ambas evaluaciones en una población de 100 encuestados. Anteriormente, [10] ya se había analizado esta comparación entre fotografías y visitas personales para comparar las evaluaciones sobre la belleza de los paisajes, observando que las evaluaciones promediadas fueron muy similares. La escasez de estos estudios hace que sea importante para futuros trabajos verificar la validez de las fotografías como medio para obtener las valoraciones del público, ya que esto proporcionará un ahorro significativo a la hora de realizar las encuestas a los ciudadanos.

Así, el objetivo de este trabajo es precisamente comparar tasaciones de arbolado urbano por parte de los ciudadanos, en su visualización "in situ" y con fotografía digital.

\section{Materiales y métodos}

\subsection{Lugar y ejemplares empleados}

Madrid es una ciudad con 3.221.824 de personas censadas y que ofrece un patrimonio verde de excepcional extensión y diversidad (más de 6.200 hectáreas), que suponen casi $20 \mathrm{~m}^{2}$ de parques y zonas verdes públicas por habitante de la ciudad. En la realización del inventario del arbolado urbano realizado en 2016 aparecen todos los árboles de jardines y pequeñas zonas verdes, así como los clasificados como parques históricos, singulares o forestales $\mathrm{y}$, también, el arbolado viario de todas las calles que componen el plano de la ciudad de Madrid. Más del 55\% de sus calles están arboladas con casi 241.000 ejemplares de 210 especies distintas.

Se ha determinado que las 10 especies de árboles más abundantes dentro del arbolado urbano de Madrid, según los datos cuantitativos del inventario de 2016 son: Acer negundo, Aesculus hippocastanum, Cupressus sempervirens, Pinus halepensis, Pinus pinea, Platanus $x$ 


\section{CONGRESO IBÉRICO DE AGROINGENIERÍA \\ X CONGRESSO IBÉRICO DE AGROENGENHARIA \\ 3 - 6 septiembre 2019, Huesca - España}

hybrida, Populus alba var. bolleana, Prunus cerasifera var. pissardii, Robinia pseudoacacia y Ulmus pumila.

Los ejemplares escogidos para la toma de datos de campo se seleccionaron de manera aleatoria en diferentes zonas de la ciudad de Madrid. Los individuos presentaban un porte adecuado, estaban situadas en lugares de fácil acceso y medianamente concurridos, ya que las encuestas debían ser realizadas en el mismo lugar donde estaba situado el árbol. Además, como debían realizarse fotografías de todos los ejemplares, se eligieron relativamente aislados (siempre que fuese posible) para evitar factores ajenos en la fotografía que pudieran influenciar de una forma u otra a la valoración.

Las localizaciones de los ejemplares fueron: el Parque de El Retiro en el centro, el Parque del Oeste, el Parque del Templo de Debod, Parque Norte, Parque de la Ventilla, Jardines del Canal de Isabel II, Barrio de El Pardo y Barrio de Montecarmelo, todos ellos en la zona norte de Madrid, Plaza de Tirso de Molina, Plaza de Las Descalzas, Calle Desengaño y Calle Carbonero y Solen para la zona centro.

\subsection{Fotos y métodos de valoración}

A cada ejemplar se le tomaron varias fotografías con cámara digital, en la época en que todos los ejemplares mostraban abundancia de hojas, con luz similar y con un encuadre que mostraba el ejemplar a completo. De ellas se seleccionó una para la encuesta, que a juicio de dos expertos en la realización de valoraciones mediante fotografía, eran las más apropiadas.

Los métodos de valoración empleados para tasar los ejemplares se describen con detalle en el trabajo de [11] y [8], reduciéndose a los tres más representativos, siendo analizados en recientes trabajos con resultados similares en [12]:

- Método Norteamericano (CTLA). Define el valor base como expresión del precio unitario de vivero según la sección del tronco.

- Método Burnley (Burnley). Utiliza como variable principal el tamaño del árbol como el volumen de un cono invertido, considerando para ello la altura y la superficie de copa.

- Norma Granada (NG). Este método utiliza la medida de la circunferencia del tronco (a $1 \mathrm{~m}$ del suelo).

Todos ellos multiplican los valores base por coeficientes correctores que incluyen factores como el estado sanitario, la especie o la localización.

Los métodos empleados requieren medir en los 100 ejemplares las siguientes variables:

Diámetro normal $(\mathrm{d})$ : en $\mathrm{cm}$, medido sobre el tronco, con corteza, a una altura de 1,30 m sobre el nivel del suelo mediante forcípula o cinta $\pi$ en los casos en los que el diámetro era tal que se superaba la escala del instrumento anterior. De esta medición se obtuvo posteriormente el perímetro o circunferencia normal.

Altura total (h): distancia entre la base del árbol y el extremo superior de la copa sobre el eje del mismo. Medido con clinómetro Suunto ó Vertex.

La variable superficie de copa (sc) se midió a posteriori en los individuos de la muestra, considerada como el ancho de la copa, la proyección de su superficie sobre el suelo en $\mathrm{m}^{2}$. Además, como la mayoría de las copas proyectan formas irregulares, se tomaron dos medidas, la de mayor longitud y su perpendicular [13], [14] con una cinta métrica y un clinómetro Suunto para comprobar la verticalidad de la posición tomada para la medición.

Las variables calculadas a partir de las variables medidas fueron:

La superficie de la copa como el área del romboide que forman las dos mediciones

Perímetro o circunferencia normal (p): en $\mathrm{cm}$, a partir del diámetro

Área de la circunferencia normal (ac): en $\mathrm{cm}^{2}$, como la superficie de la intersección del fuste con un plano perpendicular al tronco, tomada a una altura de $1,30 \mathrm{~m}$ sobre el nivel del suelo. Aunque las secciones son irregulares, se asemeja a una elipse $u$ otras figuras, para facilitar los cálculos se ha supuesto que la sección es circular. Se obtiene mediante la ecuación siguiente: 
X CONGRESO IBÉRICO DE AGROINGENIERÍA

X CONGRESSO IBÉRICO DE AGROENGENHARIA

3- 6 septiembre 2019, Huesca - España

$$
a c=\frac{\pi \cdot d^{2}}{4}
$$

Edad (t): en años, como el número de años transcurridos desde la germinación de la semilla (desde la brotación de estacas para especies de reproducción vegetativa) hasta el momento en que se realiza la determinación. Los inventarios de arbolado de las ciudades no reflejan la edad del árbol, a pesar de que sería sencillo incluir esta variable en los registros mediante el año de plantación del ejemplar y la edad indicada por el vivero. Sólo se recoge la clasificación de los ejemplares en joven maduro o viejo, por lo que es preciso estimarla.

En este caso, se empleó la ecuación del modelo de regresión lineal, obtenida con datos del arbolado de Santiago del Estero [15], debido a la imposibilidad de encontrar datos registrados de edad para ningún ejemplar.

$$
\mathrm{t}=0,701774 \mathrm{p}+0,368309 \mathrm{~h}-0,0295051 \mathrm{sc}
$$

\subsection{Realización de la encuesta}

De cada árbol muestreado se realizaron 10 encuestas a personas elegidas al azar. Con las encuestas buscamos caracterizar la población en grupos social-demográficos, como puede verse más adelante.

Después de las preguntas personales: sexo, edad, profesión, estado laboral actual y experiencia forestal, se preguntó por la preferencia en los valores económicos para cada individuo

Se preguntó cuál se ajustaba más, según su impresión, al valor real del árbol mediante una fotografía presentada en un iPad mini 4. La persona podía aumentar el tamaño de la fotografía para poder visualizarla mejor. Una vez respondían a la pregunta, les presentaba el individuo "in situ", donde volvían a responder a la pregunta de cuál era en su opinión el valor económico del árbol.

Para poder realizar ambas preguntas a las mismas personas, se ha tenido en cuenta que la realización de la encuesta fuese en un lugar próximo al ejemplar muestreado.

Para cada árbol se anotó el valor económico obtenido mediante las fórmulas de cada método, y siempre se ordenaron de la siguiente forma:

Opción 1: Método americano o CTLA

Opción 2: Norma Granada

Opción 3: Método Burnley

En ningún momento a las personas encuestadas se les dijo el nombre de los métodos de valoración, simplemente se les pidió que eligieran uno de los 3 valores económicos.

\subsection{Análisis estadístico}

Se analizan los datos desde el punto de vista descriptivo, con test de la Chi-cuadrado para detectar independencia de las variables y test de la t para proporciones. En todas las pruebas de hipótesis se ha utilizado un nivel de significación del 5\%.

Se utilizó el programa Statgraphics Centurión XVII.

\section{Resultados}

\subsection{Características de los encuestados}

El porcentaje de mujeres es de un $51 \%$ frente a un $49 \%$ de hombres. La distribución de la muestra en función de los rangos de edad que la mayor frecuencia se encuentra en personas 
entre 41 y 65 años, seguido muy de cerca por el rango de edad de los 26 a los 40 años, como se muestra en la figura 1.

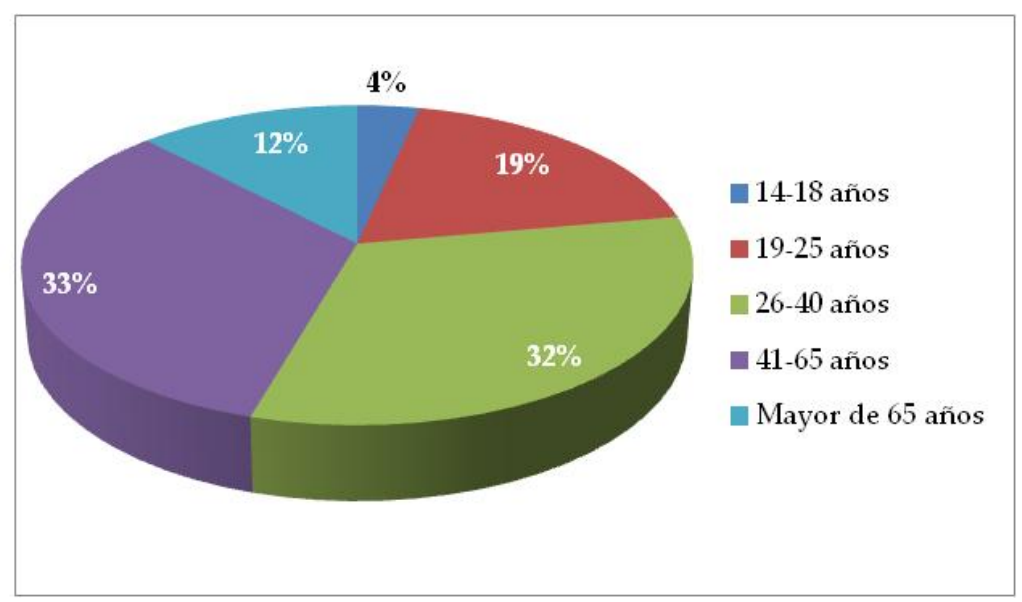

Figura 1. Distribución de la edad de los encuestados.

En función del nivel de estudios: La distribución de los encuestados en relación al nivel de estudios señala que el $64,2 \%$ de los encuestados dispone de estudios superiores y el 13,4\% tiene un nivel de estudios primarios.

En cuanto a la situación laboral en la que se encuentran, se puede indicar que el mayor porcentaje corresponde a personas que se encuentran trabajando y su distribución se muestra en la figura 2.

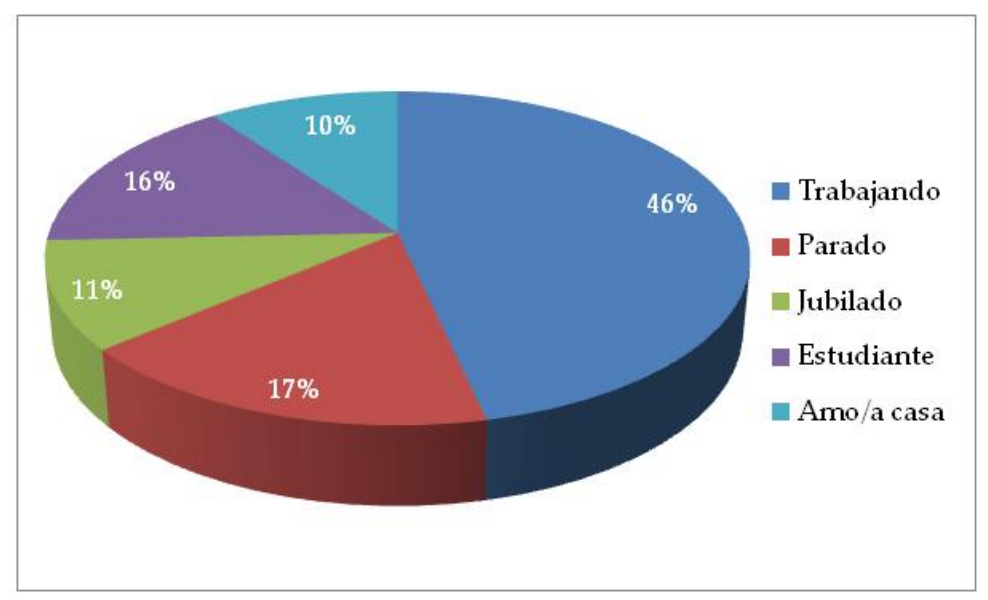

Figura 2. Distribución de la situación laboral de los encuestados.

Ninguno de los encuestados se dedica o está relacionado con una profesión forestal. Este dato es importante, ya que no se buscan valoraciones de personas con experiencia en la tasación del árbol.

\subsection{Valores de los ejemplares}

Se muestra a continuación el resumen descriptivo de los ejemplares (tabla 1) y los valores económicos obtenidos con los tres métodos (tabla 2), con los valores de la media, desviación estándar (DS), coeficiente de variación (CV), máximo y mínimo valor. 
X CONGRESO IBÉRICO DE AGROINGENIERÍA

X CONGRESSO IBÉRICO DE AGROENGENHARIA

3 - 6 septiembre 2019, Huesca - España

Tabla 1. Resumen estadístico de las medidas de los ejemplares

\begin{tabular}{cccccc}
\hline Variable & Media & DS & CV & Mínimo & Máximo \\
\hline Diámetro $(\mathrm{cm})$ & 32,32 & 12,85 & $39,78 \%$ & 11,46 & 118,47 \\
Perímetro $(\mathrm{cm})$ & 101,47 & 40,36 & $39,79 \%$ & 36,0 & 372,0 \\
Altura $(\mathrm{m})$ & 15,44 & 5,94 & $38,50 \%$ & 5,5 & 41,7 \\
Sup.copa $\left(\mathrm{m}^{2}\right)$ & 67,18 & 44,52 & $66,27 \%$ & 6,7 & 384,41 \\
Edad ajustada & 76,03 & 29,77 & $39,16 \%$ & 27,0 & 270,0 \\
\hline
\end{tabular}

Se observa una variabilidad similar de todas las medidas, exceptuando la superficie de copa, con CV el doble que el resto. Los ejemplares varían de 27 a 270 años de edad, con una edad media estimada de 76 años. La altura media es de 15 metros y el diámetro normal de 32 $\mathrm{cm}$.

Tabla 2. Resumen estadístico de valores económicos en euros

\begin{tabular}{cccccc}
\hline Variable & Media & DS & CV & Mínimo & Máximo \\
\hline CTLA & 516,99 & 577,18 & $111,64 \%$ & 99 & 4829 \\
NG & 3279,3 & 3889,64 & $118,61 \%$ & 290 & 23325 \\
Burnley & 2350,4 & 2914,21 & $123,99 \%$ & 264 & 24929 \\
\hline
\end{tabular}

Se observa una variabilidad similar en los tres métodos, siendo el CTLA el método que proporciona menores valores económicos de estos ejemplares, con la NG como método de mayor valor de tasación.

\subsection{Resultado de la encuesta}

Los resultados de la encuesta muestran que el método más seleccionado es el Burnley, seguido de la NG (figura 3)

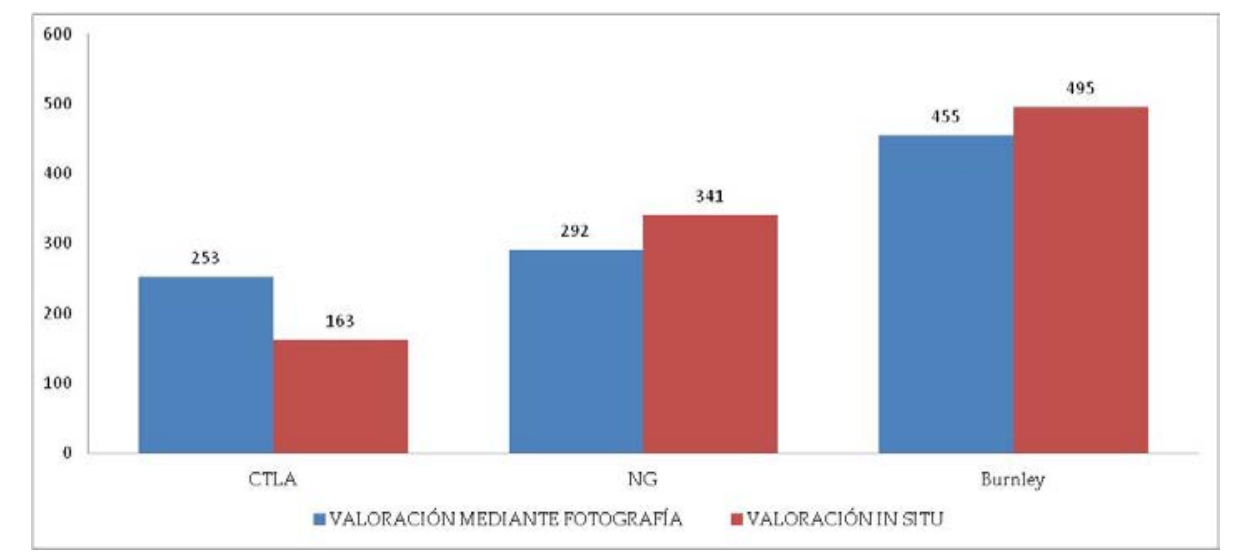

Figura 3. Comparación de valores escogidos con fotografía y al natural

Para homogeneizar los resultados se codificaron los resultados, que toman valores: -1 si el valor elegido con la fotografía superó el valor elegido "in situ", 0 cuando se eligió el mismo valor, 1 si el valor elegido "in situ" superó el valor elegido con la fotografía. Los resultados en porcentaje de respuestas se muestran en la figura 4. 


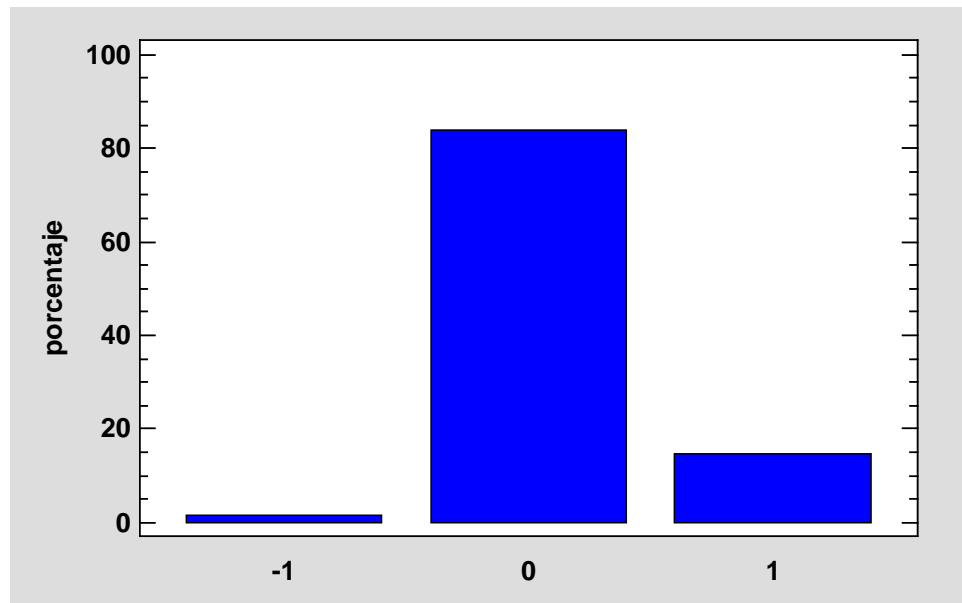

Figura 4. Porcentaje de encuestados según la diferencia de valoración realizada

El 83,8\% de las selecciones de valor coincidieron en fotografía y al natural. El 14,7\% de las elecciones "in situ" fue de valores más altos que el elegido por fotografía. Sólo un 1,5\% corresponde a una elección de valores inferior al natural que en fotografía.

Considerando los tres valores de la diferencia codificada igualmente probables (valor elegido al azar) se realiza un test para proporciones con un 95\% de confianza. La hipótesis nula es que la proporción de personas que eligieron un valor del árbol "in situ" superior al elegido con la fotografía (valor 1) es superior al 33,3\%. El resultado del test es que se rechaza la hipótesis nula con p-valor igual a 0 .

Consideramos también la hipótesis de que el valor elegido "in situ" es igual al elegido con la fotografía en un porcentaje de casos superior al elegido al azar, es decir mayor del 33,3\%. El resultado del test es que se puede admitir que la proporción es superior al 33,3\% con un $95 \%$ de confianza $(\mathrm{p}=1)$.

Por otro lado, analizando los resultados por especie de la tabla 3 muestran que las especies con mayor número de elecciones de valor superiores en los ejemplares "in situ" son el Aesculus hippocastanum, el Platanus x hybrida, seguido por Populus alba bolleana.

La prueba Chi-Cuadrado de independencia permite comprobar que existen diferencias significativas entre la elección por especie $(\mathrm{p}=0)$.

Tabla 3. Frecuencia de las diferencias según la especie

\begin{tabular}{cccc}
\hline Especie & $\mathbf{- 1}$ & $\mathbf{0}$ & $\mathbf{1}$ \\
\hline Acer negundo & 10 & 79 & 11 \\
Aesculus hippocastanum & 0 & 68 & 32 \\
Cupressus sempervirens & 0 & 94 & 6 \\
Pinus halepensis & 0 & 92 & 0 \\
Pinus pinea & 1 & 89 & 0 \\
Platanus x hybrida & 4 & 69 & 27 \\
Populus alba boll. & 0 & 80 & 20 \\
Prunus cerasifera & 0 & 90 & 10 \\
Robinia pseudoacacia & 0 & 82 & 18 \\
Ulmus pumila & 0 & 95 & 5 \\
Total por Columna & 15 & 838 & 147 \\
\hline
\end{tabular}

En el caso más extremo se puede contabilizar un total de 32 ejemplares de Aesculus hippocastanum con valores de 1 de los 100 evaluados. Y para ejemplares de Platanus x hybrida, 4 individuos con valores -1 y 27 con valores 1 . 


\section{CONGRESO IBÉRICO DE AGROINGENIERÍA \\ X CONGRESSO IBÉRICO DE AGROENGENHARIA \\ 3- 6 septiembre 2019, Huesca - España}

Para estas dos especies, no se rechaza la hipótesis nula de que la proporción de casos en que el valor elegido "in situ" es superior al elegido con la fotografía es superior al 33,3\% con un $95 \%$ de confianza ( $\mathrm{p}=0,1079$ para el $P$. hybrida y $\mathrm{p}=0,4372$ para el $A$. hippocastanum).

Analizando estos casos se observa que corresponden la mayoría a dos ejemplares de $P$. hybrida y a otros dos de A. hippocastanum pero no se ha encontrado características especiales en estos ejemplares, salvo uno de los plátanos que es un ejemplar de más de unos 200 años de edad.

Los test de la Chi-cuadrado para contrastar la hipótesis de independencia de los resultados de diferencias en la elección de valor frente a las características sociales de los encuestados no permiten rechazar la hipótesis nula con nivel de confianza del $95 \%$ ( $p=0,4$ para el sexo; $p=0,9$ para la edad; $\mathrm{p}=0,3$ para el nivel de estudios; $\mathrm{p}=0,8$ para la ocupación).

\section{Conclusiones}

Considerando el conjunto de especies analizadas se puede concluir que las personas eligen el mismo valor económico viendo los árboles urbanos tanto "in situ" como en fotografía.

Las especies P. hybrida y A. hippocastanum pueden considerarse diferentes, al menos para cuatro de los ejemplares evaluados, que se han valorado mejor "in situ" que en fotografía por la mayoría de los encuestados. En estas especies sería conveniente aumentar el número de ejemplares y de encuestados para ver si se confirma la diferencia detectada en ellos.

Por último se puede concluir que ni el sexo, la edad, el nivel de estudios o la ocupación parecen influir en las diferencias en cuanto a elección de valor.

\section{Referencias}

1. Plan de Infraestructura Verde y Biodiversidad (2017)

2. https://www.madrid.es/portales/munimadrid/es/Inicio/Medio-ambiente/Parques-y-jardines/Plan-deInfraestructura-Verde-y-

Biodiversidad/?vgnextfmt=default\&vgnextoid=5fdec0f221714610VgnVCM2000001f4a900aRCRD\&vg nextchannel=2ba279ed268fe410VgnVCM1000000b205a0aRCRD

3. Acceso 13/Mayo/2019

4. Morcillo-San Juan, A., Bautista Carrascosa, N., Borrajo Millán, J. M. (2019). Valor del bosque urbano de Madrid. Revista PARJAP 92: 24-31.

5. Ponce-Donoso, M., Vallejos-Barra, O., Daniluk- Mosquera, G. (2012). Comparación de fórmulas chilenas e internacionales para valorar el arbolado urbano. Revista Bosque 33: 69-81.

6. Caballer V. (1999). Valoración de árboles frutales, forestales medioambientales y ornamentales. Ediciones Mundi-Prensa, Madrid.

7. Cañas, I., Ayuga, E., Ayuga, F. (2009) A contribution to the assessment of scenic quality of landscapes based on preferences expressed by the public. Land Use Policy 26 (2009) 1173-1181.

8. Notaro, S., de Salvo, M. (2010). Estimating the economic benefits of the landscape function of ornamental trees in a sub-Mediterranean area. Urban Forestry and Urban Greening 9(2): 71-81.

9. Wang, R., Zhao, J. (2017). Demographic groups differences in visual preference for vegetated

10. landscapes in urban green space. Sustainable Cities and Society, 28, 350-357.

11. DOI: $10.1016 /$ j.scs.2016.10.010.

12. García-Ventura, C., Sánchez-Medina, Á., Grande-Ortíz, M., González-García, C., \& Ayuga-Téllez, E. (2018). Comparison of the Economic Value of Urban Trees through Surveys with Photographs in Two Seasons. Forests, 9(3), 132

13. Harding S.P., Burch S.E., Wemelsfelder F. (2017). The Assessment of Landscape Expressivity: A Free Choice Profiling Approach. PLoS ONE 12(1): e0169507. doi:10.1371/journal.pone.0169507.

14. Hull, R. B., \& Stewart, W. P. (1992). Validity of photo-based scenic beauty judgments. Journal of Environmental Psychology, 12(2), 101-114. 


\section{CONGRESO IBÉRICO DE AGROINGENIERÍA \\ $X$ CONGRESSO IBÉRICO DE AGROENGENHARIA \\ 3 - 6 septiembre 2019, Huesca - España}

15. Grande-Ortiz, M.A., Ayuga-Téllez, E., Contato-Carol, M.L. (2012). Methods of Tree Appraisal: A Review of Their Features and Application Possibilities. Arboriculture \& Urban Forestry 2012. 38(4): 130-140.

16. García-Ventura, C. (2013). Comparación de métodos de valoración de arbolado urbano y su aplicación al Arboreto de la ETSI de Montes (Madrid). Proyecto Fin de Carrera. Universidad Politécnica de Madrid. Madrid.

17. Thren M. 1993. Dasometría. Serie técnica Forestal. Vol. 1. Santiago del Estero, Argentina. Proyecto GTZ/UNSE, Facultad de Cs. Forestales, UNSE. 250 p.

18. Diéguez Aranda, U., Barrio Anta, M., Castedo Dorado, F., Ruiz Gonzalez, A.M., Alvarez Taboada, M.F., Alvarez Gonzalez, J.G., Rojo Alboreca, A., (2003). Dendrometría. Coedición Fundación Conde del Valle Salazar, Ediciones Mundi Prensa. 327 pp.

19. Sánchez-Medina, A., Ayuga-Téllez, E., Contato-Carol, L., Grande-Ortiz, M.A., Gonzalez-Garcia, C. (2017). Selection of Tree-Size Variables for Appraisal Methods for Urban Trees According to Their Collinearity. Arboriculture \& Urban Forestry 43 (3). 